\title{
Analysis of Cryptocurrencies Price Development
}

\author{
Jan Lánský*
}

\begin{abstract}
Cryptocurrencies are a type of digital currencies based on cryptography principles. Cryptocurrencies are a unique combination of three characteristics: they provide anonymity, they are independent of central authority and they provide protection from double spending attack. The aim of this paper is to capture trends in the area of significant cryptocurrencies price developments and to explain their causes. The current research in this area is exclusively limited to an analysis of the price developments of the most important Bitcoin cryptocurrency; our research is the first to focus on other cryptocurrencies too. The economic perspective on cryptocurrencies is based on IT knowledge regarding the principles of their functioning. We have created a database of prices of 1278 cryptocurrencies from 2013 to 2016. This database is publicly available. To analyse the data, SQL query language was used.
\end{abstract}

Keywords: Cryptocurrency, Altcoin, SQL, Price development, Price bubble.

\section{Introduction}

Cryptocurrencies are a type of digital currencies based on cryptography principles. Cryptocurrencies are a unique combination of three properties: they provide anonymity, they are independent of central authority and they provide protection from double spending attack. This combination of properties is not possessed by any other group of currencies including fiat currencies. Cryptocurrencies provide a wide range of new opportunities, but they also raise some issues. The research of cryptocurrencies is conducted in the areas of IT, economy and law. This paper tries to support the economic perspective of cryptocurrencies by IT knowledge of the problem.

Cryptocurrency research was started by Chaum (1983), when he drew up the first digital currency system. Chaum et al. (1988) summarises this into several more articles, where various other individual deficiencies of the initial draft of the system were addressed, notably those to ensure anonymity and double spending attack protection. Back (2002) came up with the proof of work concept, which was originally designed to protect email communication against spam. Haber and Stornetta (1997) drew up a data structure that is a predecessor of the blockchain structure, used in cryptocurrencies. The combination of this knowledge gave rise to Bitcoin by Nakamoto (2008), the first ever cryptocurrency, which came into existence on 3 January 2009.

\footnotetext{
* Department of Computer Science and Mathematics, Faculty of Economic Studies, University of Finance and Administration, Estonská 500, 10100 Prague 10, Czech Republic

$\triangle$ zizelevak@gmail.com
} 
The papers examining the price developments and other economic aspects are limited to the most common Bitcoin cryptocurrency. According to Coinmarketcap (2016), the market capitalization of Bitcoin amounts to $80 \%$ of the market capitalization of all cryptocurrencies. In total, the remaining approx. 600 cryptocurrencies amount only to $20 \%$ of the market capitalization, which is probably the reason why other cryptocurrencies are neglected. In this paper, we will not examine Bitcoin, but rather focus on other cryptocurrencies. With the exception of Bitcoin, for all other cryptocurrencies, the term altocoin is used in the IT field. Our paper will use more general term for a cryptocurrency so that the paper can be searched more easily even for an economically oriented reader.

The first objective of the paper is to create a database containing prices of cryptocurrencies over a comprehensive time period. The second objective is to give answers to the following research questions:

- What is the structure of cryptocurrencies by the length of their existence?

- Which cryptocurrencies experienced the highest drops in prices?

- What were the causes of the most important drops in prices of cryptocurrencies?

- Which cryptocurrencies experiences the highest price increases?

- What were the causes of the most important increases in prices of cryptocurrencies?

Chapter 2 gives an overview of literature. The research regarding the problem of cryptocurrencies is made from economic, IT and legal perspective. Chapter 3 gives an overview of our research process. First, we obtained data with prices of cryptocurrencies from (2016) in the period from 28 April 2013 to 3 July 2016. Subsequently, we adjusted the data from inconsistencies that came to existence when the cryptocurrencies were renamed. We set up a methodology for the determination of the highest price drops and increases based on the criteria of market capitalization, minimum, maximum and current price. Chapter 4 gives an overview of tour research results: overview of the existence of both active and extinct currencies, overview of the biggest slumps of cryptocurrency prices and overview of the highest increases of cryptocurrency prices. 10 cryptocurrencies with the biggest price drops and 10 cryptocurrencies with the highest price increases are dealt with in more detail. For each of these cryptocurrencies, we analysed their properties and reasons that were responsible for such significant price fluctuations. In Chapter 5, we generalized the facts learned with regard to each cryptocurrency. Chapter 6 provides an overview of the most important results of our work.

\section{Literature review}

From the economic perspective, the degree of similarity of cryptocurrencies and fiat currencies, factors having an impact on Bitcoin and price volatility of Bitcoin are most frequently dealt with. Kim (2015) analysed currencies used in massively multiplayer online role-playing games (MMORPG) and found out that they have a comparable price volatility to Bitcoin. Dwyer (2015) dealt with the analyses of demand for cryptocurrencies, the use of cryptocurrencies as the medium of exchange and analyses of the price and volatility of Bitcoin from 2010 to 2014. Using econometric tools, Cheah and Fry (2015) analysed the price of Bitcoin from 2010 to 2014. The authors demonstrated that the prices of Bitcoin has a tendency to crate bubbles. 
Ciaian et al. (2016) examined the influence of different factors on the price of Bitcoin. The factors were divided in three groups: market forces of BitCoin supply and demand, macrofinancial developments and attractiveness of Bitcoin for users. Market forces of BitCoin supply and demand were measured by the number of totally mined units of Bitcoins, by the daily number of transactions and number of addresses used on a given day. Macro-financial development was measured using oil price and the Dow Jones stock market index. The attractiveness of Bitcoin for the user was measured by the number of new comments and the number of new users on the biggest online Bitcoin forum - Bitcointalk (2016) - and the number of views of the term Bitcoin and Wikipedia. The authors managed to confirm the influence of market forces of BitCoin supply and demand and attractiveness of Bitcoin for users on the price of Bitcoin, the influence of macro-financial developments was not confirmed.

Using statistical methods, Ciaian et al. (2016) discovered that Bitcoin price developments can be divided in two periods that have different characteristics. The first period ran from the establishment of Bitcoin in January 2009 until September 2013. The second period starts in October 2013 and continues to run until present.

Dyhrberg (2016) analysed the volatility of Bitcoin using GARCH. The author found out that Bitcoin combines the properties of the US dollar and gold. Bitcoin can be used as the medium of exchange and at the same time, it is a suitable instrument for retaining the value for conservative investors. In contrast to the majority of other papers, this paper underlines the benefits of Bitcoin, while most of other papers in particular, warn of its risks. More specifically, a stark contrast can be seen in comparison with the paper of Cheah and Fry (2015). This contrast can be explained by fast development of the situation in he area of cryptocurrencies where major changes can be encounter in a single year.

From the IT perspective, anonymity, security, suitability of individual technical parameters (block site, block mining time, mining algorithm selection) and the increased usability of cryptocurrencies in real life are dealt with most frequently. Reid and Harrigan (2013) proposed a procedure for tracing the current owner of the financial means deposited in the cryptocurrency on the entered address. The procedure was based on the analysis of transaction charts and publicly available information associated with individual addresses.

Krol et al. (2013) analysed potential behaviours during the block mining. They introduced of the concept of Goldfinger attack that may result in the destruction of the specific cryptocurrency. The authors made a theoretical calculation, under which circumstances the profit for the attackers would be higher than the costs incurred on the destruction by him. Miers et al. (2013) proposed a new cryptocurrency called Zerocoin that would achieve a higher degree of anonymity of transactions than the one achieved by Bitcoin. Bonneau et al. (2015) dealt with the stability of cryptocurrency mining using mining pools. They analysed potential attacks to the mining process. They analysed risks for cryptocurrency users. The compiled an overview of alternative mining methods and approaches to anonymity. From legal perspective, the regulation of cryptocurrencies and abuse of cryptocurrencies for criminal activities is dealt with most frequently.

The European Banking Authority (2014) defined 70 risks that the use of cryptocurrencies may pose. Dual risk classification was made. The first classification was made by severity of the risk, and the second classification by the target group jeopardized by the given risk.

The New York State Department of Financial Services (2015) proposed a legal standard called BitLicence that responds to cryptocurrencies in the territory of New York. Dostov and Shust (2014) dealt with the anonymity of transactions made using Bitcoin and issues raised by 
this anonymity for the application of the rules of Anti-money laundering and combating financing of terrorism.

\section{Research methods}

Our research was conducted in three phases. In the first phase, we obtained data of daily prices of cryptocurrencies. In the second phase, the adjustment of the obtained data was made. In the third phase, we dealt with the selection of evaluation system for the most and least successful cryptocurrencies. We dealt with the length of existence of the cryptocurrency and the relation of their current price to their historical maximum and minimum. For selected cryptocurrencies, we examined the reasons of their failure or success by analysing their properties and history.

\subsection{Data collection}

First, we had to obtain data regarding the prices of cryptocurrencies. Web Coinmarketcap (2016) provides an overview of the current prices and daily transaction volumes for more than 600 cryptocurrencies. Web Coinmarketcap tries to keep record of all cryptocurrencies traded in any of more than 100 cryptocurrency exchanges. Web Coinmarketcap also offers historical snapshots starting from 28 April 2013 with seven day frequency. For the purposes of this paper, we used the data since the beginning of their availability, from 28 April 2013 to 3 July 2016.

The 7-day frequency may have a negative impact on the completeness of our results, however the data with higher precision for all existing cryptocurrencies are not available. With regard to a wide range of cryptocurrencies, there are price increases and reduction of tens of percent per day. Particularly, with newly emerging cryprocurrencies the price volatility is high. Therefore, during a single week there may be a rocket price increase amounting to the three times the original value and in the very same week the price may drop below the starting level. Such cryptocurrency would not be determined by our research as an unsuccessful one.

Coinmarketcap website offers data in html format. For the purposes of our research, we transformed the data to a relation database containing 60917 records. Following this transformation, we worked with the data using SQL query language.

Each record in our database contains eight attributes - name, symbol, date, price, cap, volume, premined and notminable. The two attributes - name and symbol - serve to identify the cryptocurrency. Symbol is usually formed by two to five letters (most frequently by three) and usually it is an acronym of name. We will use the term name (symbol). E.g., Bitcoin's symbol is BTC, thus the term Bitcoin (BTC) is used.

Attribute date shows the date when the values price, cap and volume were measured. The attribute price shows prices of the cryptocurrency on a given day. The attribute volume provides the total of values of transactions made with the cryptocurrency at exchanges on a given date. The attribute cap shows market capitalization of the cryptocurrency on a given day. The values of price, cap and volume attributes are expressed in USD. The boolean attributes premined and notminable show the cryptocurrency type by the distribution method of the cryptocurrency among users. 


\subsection{Data clearing}

First, we dealt with unique identification of the cryptocurrency. Using a SQL query we established that the combination of attributes name and symbol is unique on any monitored day. Therefore, these two attributes may be used as a unique cryptocurrency identifier.

Subsequently, we tested whether the attributes name or symbol can be used independently as a unique identifier. For symbol $s$ the uniqueness is violated, if two names $n_{1}$ a $n_{2}$ exist and that there is record containing both $n_{1}(s)$ and record containing $n_{2}(s)$ in the database. By analogy, the uniqueness for name $n$ is violated, if two symbols $s_{1}$ a $s_{2}$ exist and that there is record containing $n\left(s_{1}\right)$, and record containing $n\left(s_{2}\right)$ in the database.

The uniqueness for both attributes name and symbol is violated in our database in several dozens of cases. These uniqueness violation cases can be divided into two groups: change in cryptocurrency identification and two different cryptocurrencies with partially duplicate identification.

The first group include cryptocurrencies with regard to which the cryptocurrency identification details were changed. The authors of the cryptocurrency made a change of its attribute name, or its attribute symbol, as the case may be. For these cryptocurrencies, the older identification details were replaced for new ones in our database.

The second group is composed of pairs of different cryptocurrencies that either have an identical attribute name and different attribute symbol or they have identical attribute symbol and different attribute name. With regard to such cryptocurrencies, both attributes name and symbol must be used simultaneously for their unique identification.

The most challenging task was to differentiate when we dealt with the renaming of the cryptocurrency and when there were two different cryptocurrencies. The procedure will be demonstrate for name $n$, for which two symbols $s_{1}$ and $s_{2}$ exist. We would like to learn whether $n\left(s_{1}\right)$ and $n\left(s_{2}\right)$ stand for the same or different cryptocurrency. Should it be decided in any of the steps that renaming of the cryptocurrency occurred or that there are two different cryptocurrencies, further steps are not taken.

1. If there were $n\left(s_{1}\right)$ and $n\left(s_{2}\right)$ at least on the same day in the database, these are two different cryptocurrencies.

2. If $n\left(s_{1}\right)$ and $n\left(s_{2}\right)$ have different value at least for one of the premined and notminable attributes, these are two different cryptocurrencies.

3. For $n\left(s_{1}\right)$ and $n\left(s_{2}\right)$ the values of price, cap, volume and date attributes are manually examined for all records found. If the values are inconsistent, these are two different cryptocurrencies.

4. For $n\left(s_{1}\right)$ and $n\left(s_{2}\right)$, home websites and discussions on Bitcointalk (2016) cryptocurrency forum are manually examined. If two different websites or two different discussions are found, these are two different cryptocurrencies. On the contrary, if a single website or discussion is found, or no website or discussion is found, it means that the cryptocurrency was renamed.

\subsection{Data evaluation}

For data evaluation, SQL query language allowing us to create quickly even complicated queries was used. 
First, we dealt with the length of existence of individual cryptocurrencies measured by the number of weeks, for which the data regarding their price are available. The seven oldest cryptocurrencies in our research are handicapped as we have available only data until 28 April 2013. We were interest if there are any differences between the extinct cryptocurrencies and active cryptocurrencies. The results are shown in Table 1.

Then, we were looking for cryptocurrencies that were significant and that experienced high price drops or surges. The cryptocurrency price drop was measured as a relation of the current price to the historically maximum price. The result was a number from the interval $[0 ; 1]$. The price increase was measured as the relation of the current price to its historically minimum price. A number from the interval $[1 ; 00]$ was the result.

If the cryptocurrencies were listed by their price drops, the first place would be taken by all extinct cryptocurrencies. When cryptocurrencies are listed by the price increase, the first places would be taken by small and insignificant currencies that are rarely traded. Therefore, we must take into account the significance of the cryptocurrency. The significance of cryptocurrency was measured by its market capitalization.

We listed the cryptocurrencies in a descending order by their market capitalization and subsequently, we selected those that experienced high price slumps or surges. We had to define what we mean by high price increase or drop. The high price volatility is common for cryptocurrencies, and the cryptocurrency may lose half of its value or its value may increase two times in a single day.

With regard to price drop, we experimented with different coefficient selection when the price drop is deemed significant by us. We considered the values $0.2 ; 0.1 ; 0.05 ; 0.02 ; 0.01$ and 0.005 , and in the end we chose 0.1 as the limit. Table 2 lists cryptocurrencies in a descending order by their market capitalization that experienced a price drop to one tenth, which is equal to 0.1 coefficient at minimum.

With regard to price increase, we experimented with different coefficient selection when the price increase is deemed significant by us. We considered the values $5 ; 10 ; 20 ; 50$; and 100, in the end we chose 10 as the limit Table 3 lists cryptocurrencies in descending order by their market capitalization that experienced at least a ten times increase, which is equal to 10 coefficient.

Then we searched for more detailed information regarding their properties and history for the first ten cryptocurrencies from Tables 2 and 3. We were particularly interested in the following: Did the cryptocurrencies bring any significant innovation when established? What was the reason and purpose for the cryptocurrency establishment? What was the character of the price increase or drop (sudden or gradual)?

Technical documentation called the white paper in this area, websites or each of the cryptocurrencies, their discussion threads at Bitcointalk forum and articles on news websites dealing with cryptocurrencies were used as sources of information.

\section{Results}

At first, the data were adjusted and then we had to discover how the cryptocurrencies were renamed and which of cryptocurrencies have an identical name or symbol attribute.

Renaming of cryptocurrencies: With regard to renaming of cryptocurrencies, there were 14 changes in the name attribute and 26 changes in the symbol attribute in total. We will describe changes that presented some complications. 
If there was a simultaneous change in both name and symbol identification attributes, this change was not discovered by the methodology applied in this paper. To discover such change, additional information was required. In the past, a significant Darkcoin (DRK) cryptocurrency was renamed to Dash (DASH). This change in identification was fortunately made by Coinmarketcap websites administrators in their summary retrospectively. Mastercoin (MSC) was renamed to Omni(OMNI).

Genstrake (GEN) experienced a gradual change in both name and symbol identification attributes. At first, the symbol was changed to G3N and the in several months, also the name was changed to G3N; newly the cryptocurrency is identified as G3N (G3N).

Blitz (BLITZ) changed its name twice, first to Blitzcoin and then to Blitzcash. Scotcoin (XCPSCO) changed its symbol twice, first to NXTSCO, then to SCOT. RibbitRewards (RBR) temporarily used Loyyal name, only to return to its original name RibbitRewards. CoffeeCoin (CFC2) ceased its activities and after a year its activities were renewed under a new symbol CFC.

Identical attributes: There are 9 pairs of cryptocurrencies with a duplicate symbol and 22 pairs of cryptocurrencies with a duplicate name. Approximately in half of the cases, both cryptocurrencies affected by such duplicity are active, in the remaining cases only a single cryptocurrency is active among the duplicate cryptocurrencies.

There is even a case of threefold use of the same symbol SPC that have been gradually used by cryptocurrencies with name SaveCoin, SpinCoin, and SpikesPrivateCoin. However, at any given time no symbol was used by more than one cryptocurrency, others did no longer exist or have not yet existed at the given time. There is also a case of a threefold use of the same name Pandacoin that was used by cryptocurrencies with symbols PANDA, PAND, and PND at one time. Ghostcoin (GHC) has both duplicate name with Ghostcoin (GHOST) and duplicate symbol with GamerholicCoin (GHC).

There is also an interesting case of three cryptocurrencies InstaMineNuggets( $\$$ MINE), InstaMineNuggets A (\$MINEW), and InstaMineNuggets B (\$MINEZ) that were created by the same author at the same time and also became extinct around the same time. These cryptocurencies had a very similar name and symbol.

\subsection{Structure of cryptocurrencies by the length of their existence}

In this Chapter, we will respond to the first significant question: What is the structure of cryptocurrencies by the length of their existence? Table 1 provides a summary of the length of existence of the cryptocurrencies, in addition, the results are broken down by extinct cryptocurrencies and current cryptocurrencies. WEEKS column shows the range of the length of existence of the cryptocurrency. Each interval is equal to 25 weeks, which is approx. 6 months. ALL column represents all cryptocurrencies, being a sum of EXTINCT and ACTIVE columns. The table shows two interesting results.

From the total of 1278 cryptocurrencies, 688 are extinct, which is more than half of the total number of cryptocurrencies. More than half of the extinct cryptocurrencies became extinct within 24 weeks of their existence. On the contrary, only 3 cryptocurrencies became extinct after they survived 124 weeks of their existence and 21 cryptocurrencies became extinct from 100 to 124 weeks of their existence. There are 64 active cryptocurrencies that exist more than 124 weeks and other 107 active cryptocurrencies that exist 100 - 124 weeks.

With an increasing time of the cryptocurrency existence, the risk of its extinction is reduced, and the cryptocurrencies existing longer than 124 weeks almost never become extinct. 


\begin{tabular}{|l|rrr|}
\hline WEEKS & ALL & EXTINCT & ACTIVE \\
\hline $150-174$ & 24 & 1 & 23 \\
$125-149$ & 43 & 2 & 41 \\
$100-124$ & 128 & 21 & 107 \\
$75-99$ & 139 & 49 & 90 \\
$50-74$ & 169 & 84 & 85 \\
$25-49$ & 234 & 132 & 102 \\
$1-24$ & 541 & 399 & 142 \\
\hline SUM & 1278 & 688 & 590 \\
\hline
\end{tabular}

Table 1. Summary of the number of cryptocurrencies by the time of their existence. Source: own data processing obtained from Coinmarketcap (2015).

\subsection{The biggest price drops of cryptocurrencies}

In this Chapter, we will respond to the second significant question: Which cryptocurrencies experienced the highest drops in prices? Table 2 lists cryptocurrencies in an ascending order by heir market capitalization that experienced drop in their current price to one tenth of their historically maximum price at minimum. The table shows the first 20 cryptocurrencies of this overview.

DATE OF MAX. column shows date when the cryptocurrency reached its maximum price. The date is in the year-month-day format. MAX. CAP. \$ column shows maximum market capitalization of the cryptocurrency. AKT. VOL. $\$$ column shows the exchange trade volume of the transactions made with the given cryptocurrency over 24 hours, data as of 3 July 2016. $M A X$. PRICE \$ column shows maximum price ever reached by the cryptocurrency. AKTUAL PRICE. \$ column shows the price of cryptocurrency as of 3 July 2016. The last AKT. / MAX. column shows the ratio of the current price to the historically maximum; the overview lists only cryptocurrencies with a value lower or equal to 0.01. All prices are given in USD.

Table 2 shows the first 20 cryptocurrencies and provides very interesting results. Six cryptocurrencies are already extinct. With PayCoin there was a drop in price to one thousandth of its maximum price.

All cryptocurrencies shown in Table 2 are traded in very small volumes. Only three cryptocurrencies are traded in daily volume exceeding $\$ 10,000$, and on the contrary, three cryptocurrencies are traded in daily volume less than $\$ 500$.

Twelve of cryptocurrencies shown in Table 2 reached their maximum price in December 2013 , seven cryptocurrencies reached their maximum price in 2014 , only one cryptocurrency reached its maximum price in 2015 , none of the cryptocurrencies reached its maximum price in 2016. 


\begin{tabular}{|c|c|c|c|c|c|c|c|}
\hline NAME & SYMB. & $\begin{array}{l}\text { DATE OF } \\
\text { MAX. }\end{array}$ & $\begin{array}{l}\text { MAX. } \\
\text { CAP. \$ }\end{array}$ & $\begin{array}{l}\text { AKT. } \\
\text { VOL. \$ }\end{array}$ & $\begin{array}{l}\text { MAX. } \\
\text { PRICE \$ }\end{array}$ & $\begin{array}{l}\text { AKTUAL } \\
\text { PRICE \$ }\end{array}$ & $\begin{array}{l}\text { AKT. / } \\
\text { MAX. }\end{array}$ \\
\hline Auroracoin & AUR & 2014-03-09 & $318 \mathrm{M}$ & 3460 & 29.99 & 0.174985 & 0.006 \\
\hline Peercoin & PPC & 2013-12-01 & $150 \mathrm{M}$ & 47027 & 7.58 & 0.421533 & 0.056 \\
\hline PayCoin & XPY & 2014-12-28 & $132 \mathrm{M}$ & --- & 10.74 & 0.008425 & 0.001 \\
\hline Omni & OMNI & 2013-12-29 & $115 \mathrm{M}$ & 1112 & 184.96 & 2.62 & 0.014 \\
\hline Namecoin & NMC & 2013-12-01 & $73869 \mathrm{~K}$ & 18269 & 9.94 & 0.404434 & 0.041 \\
\hline Aphroditecoin & APH & 2014-03-23 & $70360 \mathrm{~K}$ & --- & 3.12 & --- & 0 \\
\hline Megacoin & MEC & 2013-12-01 & $44799 \mathrm{~K}$ & 5777 & 2.13 & 0.02146 & 0.010 \\
\hline Quark & QRK & 2013-12-15 & $41750 \mathrm{~K}$ & 9342 & 0.169483 & 0.005439 & 0.032 \\
\hline SpainCoin & SPA & 2014-03-16 & $37851 \mathrm{~K}$ & --- & 1.5 & --- & 0 \\
\hline Feathercoin & FTC & 2013-12-01 & $29697 \mathrm{~K}$ & 8403 & 1.2 & 0.021438 & 0.018 \\
\hline WorldCoin & WDC & 2013-12-01 & $23951 \mathrm{~K}$ & 12373 & 0.722258 & 0.009836 & 0.014 \\
\hline BitShares PTS & PTS & 2013-12-29 & $23950 \mathrm{~K}$ & 334 & 19.7 & 0.000307 & 0 \\
\hline Primecoin & XPM & 2013-12-01 & $22417 \mathrm{~K}$ & 8564 & 6.76 & $9.60 \mathrm{E}-02$ & 0.014 \\
\hline GridPay & GRID & 2015-02-22 & $21747 \mathrm{~K}$ & --- & 0.7248 & $6.90 \mathrm{E}-05$ & 0 \\
\hline Freicoin & FRC & 2013-12-01 & $16367 \mathrm{~K}$ & --- & 0.48441 & --- & 0 \\
\hline Novacoin & NVC & 2013-12-01 & $12392 \mathrm{~K}$ & 6367 & 24.63 & 0.750691 & 0.030 \\
\hline Zetacoin & ZET & 2013-12-15 & $10160 \mathrm{~K}$ & 227 & 0.064075 & 0.002781 & 0.043 \\
\hline Infinitecoin & IFC & 2014-02-02 & $10060 \mathrm{~K}$ & 12120 & 0.000112 & $7.00 \mathrm{E}-06$ & 0.063 \\
\hline Bitleu & BTL & 2014-03-30 & $9999 \mathrm{~K}$ & --- & 0.003993 & --- & 0 \\
\hline XCurrency & $\mathrm{XC}$ & 2014-07-06 & $8081 \mathrm{~K}$ & 136 & 1.47 & 0.037564 & 0.026 \\
\hline
\end{tabular}

Table 2. Overview of cryptocurrencies that reduced their value to one tenth at maximum. Source: own data processing obtained from Coinmarketcap (2015).

Now, we will respond to the third research question: What were the causes of the most important drops in prices of cryptocurrencies? We will try to clarify reasons that resulted in their price drops for the first 10 cryptocurrencies shown in Table 2.

Auroracoin (AUR): This cryptocurrency was introduced on 4 February 2014 on Bitcointalk discussion forum by Balduro (2014). The summary of basic information regarding the cryptocurrency can be found in Auroracoin (2015) discussion contribution. Auroracoin was intended for Iceland citizens, the aim of Auroracoin was to replace Icelandic Krona as a legal tender. Auroracoin inspired many other national cryptocurrencies.

Auroracoin is premined from $50 \%$ and as such the premined portion of the cryptocurrency was distributed free of charge among Iceland citizens. The distribution took place in four phases from March to November 2014. The demand among Iceland citizens was surprisingly high, more than $10 \%$ of Iceland citizens participated at least in one of the phases. In total, approx. one half of the premined volume of the cryptocurrency was distributed $(25 \%$ of the total cryptocurrency volume). To prevent citizens of other states to claim the cryptocurrency 
free of charge, there were identity checks under Kennitala, the Iceland's state identification system. Auroracoin reached the maximum price before the first distribution phase among Iceland citizens. Following each distribution wave, the price dramatically dropped, in October 2014 the current price amounted to less than $0.1 \%$ of the maximum price.

Despite the fact that the price of Auroracoin dropped sharply, the community around Auroracoin did not collapse and it still keeps this cryptocurrency alive. In April 2015, there was even a sharp surge of the price to more than 20-times of the maximum price. For this reason, Auroracoin is in Table 3 showing the examples of he biggest cryptocurrency price surges. In spite of this sharp price increase, its current price stands at less than $1 \%$ of the maximum price. According to BBC (2016, the sharp price increase was caused by the crises on the local political scene in Iceland in connection with the Panama Papers scandal.

Peercoin (PPC): King \& Nadal (2012) introduced white paper of Peercoin. Peercoin brought an important innovation with regard to the mining mechanism. It was the first cryptocurrency using a combination of proof-of-work and proof-of-stake for mining. This innovation was the reason for the high price of the cryptocurrency in the past. At the turn of 2013 and 2014, Peercoin join the popularity wave of Bitcoin and reached its maximum price. It has been on steady decline ever since then. The newly emerging cryptocurrencies provide more extensive innovations than the ones offered by Peercoin.

PayCoin (XPY): There are two cryptocurrencies called PayCoin, this text deals with the cryptocurrency with XPY symbol. White paper of PayCoin (XPY) can no longer be found at the official website of the cryptocurrency, however it is still available on Dognose website (2016). According to Higgins (2015a), the cryptocurrency was created by GAW, a US-based mining company in December 2014. Compared to other cryptocurrencies of that time, the cryptocurrency did not offer any significant innovations. GAW was making money by renting mining capacities for mining of PayCoin (XPY). The customers were deceived by a false promise that GAW will never allow a price drop below $\$ 20$. According to Higgins $(2015 b)$, for this false promise GAW now faces civil action. PayCoin (XPY) can be classified as fraudulent.

Omni (OMNI): According to Buterin (2013a), Mastercoin (MSC) was established at the beginning of 2012, the principles of its functioning were described by Willett (2012) in the white paper. According to Rizzo (2015), the cryptocurrency was renamed to Omni (OMNI) in February 2015. Omni is a software layer built on top of the Bitcoin (BTC) blockchain. Omni serves for an easy creation and functioning of new cryptocurrencies. Significant cryptocurrencies, such as Factom (FCT), MaidSafeCoin (MAID) and Tether (USDT) use Omni for their existence.

When established, Omni (then under the name Mastercoin) was a significant innovation raising high demand among investors. At the turn of 2013 and 2014, the maximum price was reached, which was caused by a sharp surge of the price of Bitcoin. In spring 2014, the price experienced a sharp drop. Omni has currently a very low volume of transactions, and its price dropped to approx. $1 \%$ of the maximum price. At present, there are many cryptocurrencies with better functionalities. To create a new cryptocurrency using Omni, only a very small amount of Omni is sufficient. These are the two main reason of low demand for this cryptocurrency.

Namecoin (NMC): Namecoin is one of the oldest cryptocurrencies, as it was established as early as in 2011. Namecoin reached its maximum price at the turn of 2013 and 2014 when it joined the wave of an increased demand for Bitcoin. In spring 2014, the price experienced a sharp drop, and it has been on steady decline ever since then. 
Kalodner et al. (2015) describes the functionality of Namecoin and analyses its use in real life. Namecoin serves to register the ownership of text identifiers- names and web addresses in the bit Internet domain. Since the establishment of the cryptocurrency in 2011 and until $2015,120.000$ names had been registered, but only 28 of them are not squatted, and have nontrivial content. There have been also only 250 business transactions when names were transferred into ownership for a charge. The low usage in practice and existence of many competing cryptocurrencies offering better functionality are the reason for the current low price of Namecoin.

Aphroditecoin (APH): Aphroditecoin was established in March 2014 as a national cryptocurrency for Cyprus, then struck by financial crisis. At present, Aphroditecoin is already extinct, its website no longer works, however Aphroditecoin (2014) discussion on Bitcointalk forum is available. Auroracoin for Iceland served as an example for Aphroditecoin. The reasons for extinction of Aphroditecoin are similar to the reason for the loss of more than $99 \%$ from the maximum price of the national cryptocurrency for Iceland Auroracoin.

Megacoin (MEC): Megacoin was introduced in the middle of 2013 in Bitcointalk forum by Chan (2013). Megacoin did not bring any innovations and as opposed to national cryptocurrencies is intended for no specific group of users. Its price reached the maximum at the turn of 2013 and 2014, followed by sharp slump of the price in the beginning of 2014 and subsequent steady decline of the price until present. The current price amounts to $1 \%$ of the historically maximum prices. However, Megacoin did not become extinct, the community around it ensuring it marketing still exists, and there are also localizations of the Megacoin (2013) website in 20 languages.

Quark (QRK): Quark was introduced in the middle of 2013 in Bitcointalk forum by Quarkcoin (2013). For mining purposes, Quarkcoin used 6 different hash functions to eliminate the advantage of mining using Application Specific Integrated Circuit (ASIC). The aim was to return the mining to personal computers and graphics cards. The price of Quark reached its maximum at the turn of 2013 and 2014, with the current price amounting to approx. $3 \%$ of the historically maximum price.

SpainCoin (SPA): According to SpainCoinDev (2014) in Bitcointalk forum, SpainCoin was established in March 2014 as a national cryptocurrency for Spain. Currently, this cryptocurrency is already extinct.

Feathercoin (FTC): Quark was introduced in the middle of 2013 in Bitcointalk forum by Bushstar (2013). Since 2014, Neoscrypt hash function has been used for mining, with an aim to eliminate mining using ASIC. The price of Feathercoin reached its maximum at the turn of 2013 and 2014, with the current price amounting to approx. $2 \%$ of the historically maximum price.

Summary of reasons for price drops: At the turn of 2013 and 2014, there was a bubble on the cryptocurrency market. All target with the sharp increase of Bitcoin, followed by price increases of other cryptocurrencies. However, this growth had no fundamental basis. More than half of the biggest price drops was caused by correction following this event.

National cryptocurrencies represent other group of failed cryptocurrencies. The cryptocurrency was evenly distributed to citizens of a given state who showed interest in the cryptocurrency. Unfortunately, the owners of these cryptocurrencies immediately their sale which resulted in a drastic loss of value of the given cryptocurrency, or its extinction. 
The third large group of failed cryptocurrencies is constituted by cryptocurrencies that brought a technical or application innovation at that time, however they were overcome by their followers in his regard. The development in the field of cryptocurrencies is very dynamic.

\subsection{The biggest price increases of cryptocurrencies}

In this Chapter, we will respond to the fourth significant question: Which cryptocurrencies experience the highest price increases? Table 3 lists cryptocurrencies in an descending order by their market capitalization the current price of which experienced reached at least ten times of their historically minimum price. The table shows the first 20 cryptocurrencies of this overview.

\begin{tabular}{|lllrrrrr|}
\hline NAME & SYMB. & MIN. & CAP. $\$$ & VOL. $\$$ & PRICE $\$$ & \multicolumn{1}{l}{$\begin{array}{l}\text { AKTUAL } \\
\text { PRICE } \$\end{array}$} & \multicolumn{1}{l}{ MIN. } \\
\hline Bitcoin & BTC & $2013-07-07$ & $775 \mathrm{M}$ & $114 \mathrm{M}$ & 68.1 & 689.28 & 10.12 \\
Ethereum & ETH & $2015-10-18$ & $40 \mathrm{M}$ & $9541 \mathrm{~K}$ & 0.544664 & 12.04 & 22.11 \\
Dash & DASH & $2014-02-16$ & $1221 \mathrm{~K}$ & $290 \mathrm{~K}$ & 0.366146 & 7.21 & 19.69 \\
NEM & XEM & $2015-09-13$ & $813 \mathrm{~K}$ & $1078 \mathrm{~K}$ & $9.00 \mathrm{E}-05$ & 0.010495 & 116.61 \\
Synereo & AMP & $2015-12-20$ & $761 \mathrm{~K}$ & 17568 & 0.004116 & 0.072114 & 17.52 \\
\hline Factom & FCT & $2015-12-13$ & $716 \mathrm{~K}$ & $683 \mathrm{~K}$ & 0.081726 & 1.39 & 17.01 \\
Storjcoin X & SJCX & $2015-11-22$ & $450 \mathrm{~K}$ & 14833 & 0.010106 & 0.113268 & 11.21 \\
Siacoin & SC & $2015-12-27$ & $143 \mathrm{~K}$ & $531 \mathrm{~K}$ & $1.70 \mathrm{E}-05$ & 0.000804 & 47.29 \\
VeriCoin & VRC & $2014-05-25$ & 99795 & 15585 & 0.00373 & 0.060238 & 16.15 \\
Auroracoin & AUR & $2015-10-11$ & 98434 & 3460 & 0.013489 & 0.174985 & 12.97 \\
\hline SysCoin & SYS & $2015-02-22$ & 92831 & 25263 & 0.000231 & 0.00802 & 34.72 \\
Mintcoin & MINT & $2015-01-18$ & 60256 & 2247 & $3.00 \mathrm{E}-06$ & $7.10 \mathrm{E}-05$ & 23.67 \\
BoostCoin & BOST & $2015-04-19$ & 58763 & 1256 & 0.004994 & 0.075741 & 15.17 \\
VPNCoin & VPN & $2014-12-07$ & 57607 & 42999 & 0.000144 & 0.008614 & 59.82 \\
EmerCoin & EMC & $2014-08-24$ & 49616 & 69097 & 0.001504 & 0.338172 & 224.85 \\
\hline NautilusCoin & NAUT & $2015-02-01$ & 48191 & 33641 & 0.009085 & 0.41055 & 45.19 \\
EarthCoin & EAC & $2015-04-26$ & 44000 & $228 \mathrm{~K}$ & $6.00 \mathrm{E}-06$ & 0.000207 & 34.5 \\
SolarCoin & SLR & $2014-07-27$ & 39670 & 9803 & 0.001567 & 0.090845 & 57.97 \\
UnionCoin & UNC & $2015-04-12$ & 33879 & 1496 & 0.004039 & 0.067345 & 16.67 \\
Ixcoin & IXC & $2013-07-07$ & 32853 & 11 & 0.00234 & 0.046017 & 19.67 \\
\hline
\end{tabular}

Table 3. Overview of cryptocurrencies hat increased their value at least ten time. Source: own data processing obtained from Coinmarketcap (2015).

DATE OF MIN. column shows date when the cryptocurrency reached its minimum price. The date is in the year-month-day format. MIN. CAP. \$ column shows minimum market capitalization of the cryptocurrency. AKT. VOL. \$ column shows the exchange trade volume 
of the transactions made with the given cryptocurrency over 24 hours, data as of 3 July 2016. MIN. PRICE $\$$ column shows minimum price ever reached by the cryptocurrency. AKTUAL PRICE. \$ column shows the price of cryptocurrency as of 3 July 2016. The last AKT. / MIN. column shows the ratio of the current price to the historically minimum; the overview lists only cryptocurrencies with a value higher or equal to 10. All prices are given in USD.

Table 3 shows the first 20 cryptocurrencies and provides very interesting results. Twelve of the cryptocurrencies shown had the market capitalization under $\$ 100,000$ when it reached the maximum price, and only three cryptocurrencies had their market capitalization above $\$ 1,000,000$. In 2016, none of the cryptocurrencies shown, did not experience its minimum price. The biggest increase was experienced by EmerCoin, increasing its value more than 224-times. Ixcoin is seldom traded.

Now, we will respond to the fifth question of the research: What were the causes of the most important increases in prices of cryptocurrencies? We will try to clarify reasons that resulted in their price drops for the first 10 cryptocurrencies shown in Table 3. Auroracoin is also inn Table 2 and discussion regarding its price development can be found in the previous chapter. Therefore, we will only analyse 9 cryptocurrencies in this chapter.

Bitcoin (BTC): Bitcoin is the oldest cryptocurrency. On the basis of white paper drafted by Nakamoto (2008) Bitcoin was launched in January 2009. At present, Bitcoin has approx. 80\% of the market capitalization of all cryptocurrencies. Therefore, other cryptocurrencies are very often traded for Bitcoin. The data for our paper represent only a small part of the history of Bitcoin, therefore we will not evaluate this cryptocurrency more closely. However, we recommend papers that have dealt with a comprehensive price development of Bitcoin: Cheah and Fry (2015), Ciaian et al. (2016), Dyhrberg (2016) and Dwyer (2015).

Ethereum (ETH): Ethereum was introduced towards the end of 2013 in the white paper drafted by Buterin (2013b). Etherem is the first cryptocurrency which is Turing complete. This innovation allows to create smart contracts, autonomous applications stored in blockchain. In practice, Decentralized Autonomous Organization (The DAO) is the most extensive smart contracts application; however due to implementation error it was attacked by hackers shortly after its creation.

Ethereum brought many other innovations. The inclusion of uncle blocks in the blockchain allows generating blocks every 15 seconds. The dynamic fee setting for transactions limits denials of service attacks. The mining is performed through proof-of-work using an algorithm entirely eliminating the benefits of specialized ASIC against CPU and GPU.

Dash (DASH): Dash was introduced at the beginning of 2014 in the white paper drafted by Duffield, E. \& Diaz, D. (2014). The main innovation of Darkcoin is an option to anonymize transactions using the Darksend method. In classic transactions, the addresses of a sender and recipient are given. When the Darksend method are used, the addresses of senders are mixed with addresses of recipients from more different transactions, and thus the tracing of the recipient if the sender is known is made impossible. With other cryptocurrencies, the functionality provided using Darksend must be provided through an external service, called mixer. Dash was originally Darkcoin (DRK), but in 2015 it was renamed, as it was confused with illegal activities due to its original name.

NEM (XEM): NEM was introduced at the beginning of 2014 in Bitcointalk forum by Utopianfuture (2014), however not to be launched earlier than in May 2015. Cryptocurrency NEM was supposed to be by simply derivative NEXT, but finally has its own protocol written from scratch. At that time, Nxt was the most innovative cryptocurrency allowing e.g. smart contracts. Both Nxt and NEM are premined using proof-of-stake to mine blocks. While the 
original distribution of the currency of Nxt cryptocurrency was divided among 73 original investors, the founders of NEM did try to perform original currency distribution among more investors and the individual users had equal shares at the same time.

Synereo (AMP): Synereo was introduced towards the end of 2014 in Bitcointalk by Elokane (2014). The cryptocurrency is used as means of payment in the decentralized social network bearing the same name where all user data are encrypted and the users alone decide which data will be made accessible to whom and for what price. It is a contrast with classic Facebook social network where the advertising is forced upon the users and the entire profit from advertising is gained by the social network operator.

Factom (FCT): Factom was introduced towards the end of 2014 in the white paper drafted by Snow et al. (2014). The cryptocurrency serves to make queries regarding or disprove the existence of the document in the given time. The hash is calculated from the document and then it is stored in blockchain. Subsequently, at any time the document is presented for inspection, the hash is calculated from the document and if the hash is in the blockchain, then the document existed at the time when the block where the document has is located, was created. Using Factom, different services that are currently provided by government institutions, may be provided (such as document verification by notaries, register of real estates and cars).

Storjcoin X (SJCX): Storjcoin X was introduced in the middle of 2014 in Bitcointalk forum by Storj (2014), a similar specification can be found in the white paper by Wilkinson (2014). The cryptocurrency serves for a distributed document storage. The document is divided in smaller parts, these are encrypted and are redundantly stored in network nodes. The network modes are rewarded for storage of the document parts, which is paid for by the owner.

Siacoin (SC): Storjcoin X was introduced in the middle of 2015 in Bitcointalk forum by Taek (2015). Similarly as Storjcoin X, the cryptocurrency serves for a decentralized cloud document storage.

VeriCoin (VRC): VeriCoin (VRC) was introduced in the middle of 2014 in Bitcointalk forum by EffectsToCause (2014). The main motivation was to credit interest to individual amounts held by users. The cryptocurrency has a very strong marketing and is accepted as means of payment by many merchants who accept Bitcoin.

Summary of reasons for price increases: The highest cryptocurrency price increases occur when at least two of the following criteria are met. The cryptocurrency brought a significant technology innovation. The cryptocurrency offers a decentralized service demanded by users. A team of people, usually called foundation, manages the development and promotion of the cryptocurrency.

\section{Discussion}

EBA (2014) defined 70 risks arising from the ascent of cryptocurrencies. Our research confirms the existence of the risk - User experiences drop in value of VCs due to significant or unexpected exchange rate fluctuation (A03). At the turn of 2013 and 2014, Bitcoin experienced a sharp price increase followed by the price increase of other cryptocurrencies. Following the sharp price increase, sharp drop followed. Table 2 shows 20 cryptocurrencies tnat experienced the biggest price drops. Twelve of those cryptocurrencies reached their maximum prices in this period.

Our research also confirms the existence of the risk - User suffers loss when buying VCs that do not have the VC features that the user expects (A06). A detailed comment to the risk says 
that these are instances when two cryptocurrencies have a identical name attribute. During the data adjustment we established that: There are 9 pairs of cryptocurrencies with a duplicate symbol and 22 pairs of cryptocurrencies with a duplicate name. Cryptocurrencies are also being renamed, there were 14 changes in the name attribute and 26 changes in the symbol attribute in total.

In contrast, our research contradicts the existence of the risk - User suffers loss due to changes made to the VC protocol or other key components (A08). This risk did not manifest (from 28 April 2013 to 3 July 2016) for none of the cryptocurrencies that we evaluated. The changes in the protocol of cryptocurrencies occur very rarely and usually functionalities are extended not limited. None of the changed experienced have resulted in any harm incurred by the users of cryptocurrency.

Our research partially confirms the risk - User suffers loss as a result of VC prices being manipulated (A41). We examined 10 cryptocurrencies that experienced sharp price drops and at the time had high market capitalization in an extensive detail. Of these, only a single cryptocurrency - PayCoin (XPY) was fraudulent. This result can be surprising; we expected much higher number of fraudulent cryptocurrencies in the reviewed sample.

Using statistical methods, Ciaian et al. (2016) examined factors affecting the price of Bitcoin. The authors established that the attractiveness factor of Bitcoin for users have an impact on the price of Bitcoin. Our research partially confirms the finding above. Megacoin (MEC) achieved high market capitalization without bringing a new innovation or useful decentralized service. However, the cryptocurrency has a strong marketing. Even though a rather sharp drop of the cryptocurrency price followed, the cryptocurrency did not become extinct.

Auroracoin (AUR) is an attempt to introduce national cryptocurrency in Iceland. Following the sharp price drop in the middle of 2014, the value was increased ten times in April 2015 in response to the political crisis in Iceland. It would be interesting to examine whether and how political factors influence prices of cryptocurrencies.

Cheah and Fry (2015) proved that Bitcoin has a tendency to create price bubbles. Our research confirms that also other cryptocurrencies have tendencies to follow price bubbles created by Bitcoin. 12 of 20 cryptocurrencies from Table 2 showing the biggest price slumps became victims of the bubble from the turn of 2013 and 2014.

Miers et al. (2013) theoretically proposed a new cryptocurrency called Zerocoin that would achieve a higher degree of anonymity of transactions than Bitcoin. Our research proved that at least some cryptocurrency users are interest in an increased anonymity, which can be demonstrated by the success of Dash (DASH).

\section{Conclusion}

This papers of the first of its kind to deal with the analysis of cryptocurrency price development. Previous papers have exclusively dealt with the price of the most common cryptocurrency -Bitcoin. At first, we created a database with prices of cryptocurrencies and then we analysed he cryptocurrencies by their length of existence, the biggest price drops and highest price increases of cryptocurrencies.

The database created by us includes 60917 records with prices of 1278 cryptocurrencies in the period from 28 April 2013 to 3 July 2016. Historical snapshots from Coinmarketcap (2016) with a 7-day frequency were used as a source of data. The database created by us is available at http://goo.gl/efBPSW. 
Each cryptocurrency in our database is uniquely identified by a pair name and symbol. Neither the name attribute alone, nor the symbol attribute alone do provide a unique identification of the cryptocurrency. There are 9 pairs of cryptocurrencies with a duplicate symbol and 22 pairs of cryptocurrencies with a duplicate name. While adjusting the data we established that there were 14 changes in the name attribute and 26 changes in the symbol attribute in total. This renaming had to be done in our database with regard to records with old values.

We have analysed the structure of cryptocurrencies by the length of their existence; the results are given in Table 1. From all 1278 cryptocurrencies, 688 became extinct. More than half of the extinct cryptocurrencies became extinct within 24 weeks of their existence. In contrast, only 3 cryptocurrencies became extinct after surviving 124 weeks of their existence. There are 64 active cryptocurrencies that exist more than 124 weeks.

We have compiled an overview of cryptocurrencies that experienced a drop in their current price at least to one tenth to their maximum price. This overview is listed in a descending order by market capitalization of the currencies as of the date when their reached their maximum price. Table 2 shows the first 20 cryptocurrencies of this overview. With regard to the first 10 cryptocurrencies from this overview, we examined their properties and history and determined reasons of this price drop. The reasons for the price drop were following: price bubble from the turn of 2013 and 2014, failure of the concept of national cryptocurrencies and overcoming a cryptocurrency by a new cryptocurrency with higher degree of innovation. Only a single cryptocurrency that was examined in much detail was fraudulent - PayCoin (XPY).

We have compiled an overview of cryptocurrencies that experienced an increase in their current price at least to ten times of their minimum price. This overview is listed in a descending order by market capitalization of the currencies as of the date when their reached their minimum price. Table 3 shows the first 20 cryptocurrencies of this overview. The biggest appreciation was experienced by EmerCoin, increasing its value more than 224-times. With regard to the first 10 cryptocurrencies from this overview, we examined their properties and history and determined reasons of this price increase. The reasons for price increases were following: The cryptocurrency brought a significant technology innovation. The cryptocurrency offers a decentralized service demanded by users. A team of people, usually called foundation, manages the development and promotion of the cryptocurrency.

The biggest problem that new cryptocurrencies face is the initial price of the cryptocurrency. The first cryptocurrency - Bitcoin - started with no value whatsoever and its price increased with the increasing number of users. When established, new cryptocurrencies have a price artificially set by their authors. Very often, there experience sharp increases or drops.

In the future, we will continue to research the problem of cryptocurrency price developments. The theme of prediction of future cryptocurrency price developments based on the properties of this cryptocurrency is an interesting one. We would like to try to create a cryptocurrency index, being an analogy to stock index.

\section{Acknowledgement}

This research was supported by the Czech Science Foundation as part of the project New Sources of Systemic Risk on Financial Markets (GA ČR 16-21506S). 


\section{References}

Aphroditecoin (2014). Aphroditecoin introduced to boost economy and help Cypriots return to financial stability. Retrieved from: https://bitcointalk.org/index.php?topic=511160.0

Auroracoin (2015). AURORACOIN - Empowering Financial Freedom. Retrieved from: https://bitcointalk.org/index.php?topic=1044432.0

Back, A. (2002). Hashcash - A Denial of Service Counter-Measure. Retrieved from: http://www.hashcash.org/papers/hashcash.pdf

Balduro (2014). Auroracoin - a cryptocurrency for Iceland. Retrieved from: https://bitcointalk.org/index.php?topic $=446062.0$

BBC (2016). Panama Papers: Iceland PM Sigmundur Gunnlaugsson steps down. Retrieved from: https://bitcointalk.org/index.php?topic=1044432.0

Bitcointalk (2016). Bitcoin Forum. Retrieved from: https://bitcointalk.org/

Bonneau, J., Miller, A., Clark, J., Narayanan, A., Kroll, J. A. \& Felten, E. W. (2015). SoK: Research Perspectives and Challenges for Bitcoin and Cryptocurrencies. In Proceedings of the 2015 IEEE Symposium on Security and Privacy, (pp. 104-121). New York: IEEE. doi: 10.1109/SP.2015.14

Bushstar (2013) Feathercoin - time proven, fast, secure and unique POW coin. Retrieved from: https://bitcointalk.org/index.php?topic=178286.0

Buterin, V. (2013a). Mastercoin: A Second-Generation Protocol on the Bitcoin Blockchain. Retrieved from: https://bitcoinmagazine.com/articles/mastercoin-a-second-generation-protocol-on-thebitcoin-blockchain-1383603310

Buterin, V. (2013b). Ethereum White Paper. Retrieved from: https://github.com/ethereum/wiki/wiki/White-Paper

Chan, K. (2013). Megacoin has arrived. Scrypt \& Core 0.8.2+ Updates: Chinese Market. Retrieved from: https://bitcointalk.org/index.php?topic=218851.0

Chaum, D. (1983). Blind signatures for untraceable payments. In Advances in Cryptology Proceedings of Crypto 82, (pp. 199-203). New York: Springer. doi: 10.1007/978-1-4757-0602$\underline{418}$

Chaum, D., Fiat, A. \& Naor, M. (1988). Untraceable electronic cash. In CRYPTO 88 Proceedings on Advances in Cryptology, (pp. 319-327). New York: Springer. doi: 10.1007/0-387-34799-2 25

Cheah, E.-T. \& Fry, J. (2015). Speculative bubbles in Bitcoin markets? An empirical investigation into the fundamental value of Bitcoin. Economics Letters, 130, 32-36. doi: 10.1016/j.econlet.2015.02.029

Ciaian, P., Rajcaniova, M. \& Kancs, d'A. (2016). The economics of BitCoin price formation. Applied Economics, 48(19), 1799-1815. doi: 10.1080/00036846.2015.1109038

Coinmarketcap (2016). Crypto-Currency Market Capitalizations. Retrieved from: http://coinmarketcap.com/

Dognose (2016). Paycoin A cryptocurency fit for world adoption White Paper. Retrieved from: http://www.dognose.com/ paycoin/paycoin-whitepaper.pdf

Dostov, V. \& Shust, P. (2014) Cryptocurrencies: an unconventional challenge to the AML/CFT regulators? Journal of Financial Crime, 21(3), 249-263. doi: 10.1108/JFC-06-2013-0043

Duffield, E. \& Diaz, D. (2014). Dash: A Privacy-Centric Crypto-Currency. Retrieved from: https://www.dash.org/wp-content/uploads/2015/04/Dash-WhitepaperV1.pdf

Dwyer, G. P. (2015). The economics of Bitcoin and similar private digital currencies. Journal of Financial Stability, 17, 81-91. doi: 10.1016/.j.js.2014.11.006

Dyhrberg, A. H. (2016). Bitcoin, gold and the dollar - A GARCH volatility analysis. Finance Research Letters, 16, 85-92, doi: 10.1016/i.frl.2015.10.008 
EffectsToCause (2014). VeriCoin PoST Currency. Retrieved from: https://bitcointalk.org/index.php?topic $=602041.0$

Elokane (2014). Synereo: A fully decentralized social network owned by you. Retrieved from: https://bitcointalk.org/index.php?topic=827782.0

European Banking Authority (2014). EBA Opinion on virtual currencies. EBA/Op/2014/08.

Haber, S. \& Stornetta, W. S. (1997). Secure names for bitstrings. In Proceedings of the 4th ACM Conference on Computer and Communication Security, (pp. 28-35). New York: ACM. doi: $\underline{10.1145 / 266420.266430}$

Higgins, S. (2015a). SEC GAW Miners and the Disappearing \$20 Paycoin Floor. Retrieved from: http://www.coindesk.com/gaw-miners-paycoin-disappearing-20-floor/

Higgins, S. (2015b). SEC Charges GAW Miners CEO Josh Garza With Securities Fraud. Retrieved from: http://www.coindesk.com/gaw-faces-ponzi-scheme-charges-from-sec/

Kalodner, H., Carlsten, M., Ellenbogen, P., Bonneau, J. \& Narayanan, A. (2015). An empirical study of Namecoin and lessons for decentralized namespace design. In The 14th Workshop on the Economics of Information Security, (pp. 1-23). Delft: TU Delft.

Kim T. (2015). The Predecessors of Bitcoin and Their Implications for the Prospect of Virtual Currencies. PLOS ONE, 10(4), 1-18. doi: 10.1371/journal.pone.0123071

King, S. \& Nadal, S. (2012). PPCoin: Peer-to-Peer Crypto-Currency with Proof-of-Stake. Retrieved from: https://www.peercoin.net/assets/paper/peercoin-paper.pdf

Kroll, J. A., Davey, I. C. \& Felten, E. W. (2013). The Economics of Bitcoin Mining or, Bitcoin in the Presence of Adversaries. In The Twelfth Workshop on the Economics of Information Security, (pp. 1-21). Washington, D.C.: Georgetown University.

Megacoin (2013). Megacoin Your Global Currency. Retrieved from: http://www.megacoin.co.nz/

Miers, I., Garman, C., Green, M. \& Rubin, A. D. (2013). Zerocoin: Anonymous Distributed E-Cash from Bitcoin. In IEEE Symposium on Security and Privacy, (pp. 397-411). New York: IEEE. doi: 10.1109/SP.2013.34

Nakamoto, S. (2008). Bitcoin: A Peer-to-Peer Electronic Cash System. Retrieved from: https://bitcoin.org/bitcoin.pdf

New York State Department of Financial Services (2015). New York codes, rules and regulations. Title 23. Department of Financial Services. Chapter I. Regulations of the superintendent of financial services. Part 200 Virtual Currencies.

Popper, N. (2016). A Hacking of More Than $\$ 50$ Million Dashes Hopes in the World of Virtual Currency. The New York Times. Retrieved from: http://www.nytimes.com/2016/06/18/business/dealbook/hacker-may-have-removed-more-than50-million-from-experimental-cybercurrency-project.html

Quarkcoin (2013). Quark Core 0.9.2.7. Retrieved from: https://bitcointalk.org/index.php?topic=260031.0

Reid, F. \& Harrigan, M. (2013). An Analysis of Anonymity in the Bitcoin System. In Y. Altshuler at al. (Eds.), Security and Privacy in Social Networks, (pp. 197-223). New York: Springer. doi: 10.1007/978-1-4614-4139-7

Rizzo, P. (2015). Mastercoin Seeks Second Start With Omni Reboot. Retrieved from: http://www.coindesk.com/mastercoin-new-beginning-omni/

SpainCoinDev (2014). SpainCoin: freedom for Spain - Airdrop ended, last coins destroyed!! Retrieved from: https://bitcointalk.org/index.php?topic $=500511.0$

Snow, P., Deery, B., Lu, J., Johnston, D. \& Kirby, P. (2014). Factom Business Processes Secured by Immutable Audit Trails on the Blockchain. Retrieved from: https://raw.githubusercontent.com/FactomProject/FactomDocs/master/Factom Whitepaper.pdf

Storj (2014). Storjcoin X (SJCX) - A Coin for Decentralized Cloud Storage. Retrieved from: https://bitcointalk.org/index.php?topic=702602.0 
Taek (2015). Sia - Decentralized Storage - Working Beta - Currency has been released. Retrieved from: https://bitcointalk.org/index.php?topic=1060294.0

Utopianfuture (2014). NEM -New Economy Movement - No Envy Movement - Updates+Discussion thread. Retrieved from: https://bitcointalk.org/index.php?topic=426303.0

Wilkinson, S. (2014). Storj A Peer-to-Peer Cloud Storage Network. Retrieved from:

https://stori.io/stori.pdf

Willett, J. R. (2012). The Second Bitcoin Whitepaper. vs. 0.5. Draft for Public Comment. Retrieved from: https://sites.google.com/site/2ndbtcwpaper/2ndBitcoinWhitepaper.pdf 
